\title{
Nonlinear Response Characteristics of Giant Magnetostrictive-Piezoelectric Composite Sensors
}

\author{
Jia $\mathrm{Xu}^{1,2}$, Ranzhen $\mathrm{Li}^{1}$ and Zhiwen $\mathrm{Zhu}{ }^{1, *}$ \\ 'Department of Mechanics, Tianjin University, Tianjin 300072, China \\ ${ }^{2}$ Tianjin Key Laboratory of Nonlinear Dynamics and Chaos Control, Tianjin 300072, China
}

(Received July 2, 2014; accepted April 2, 2015)

Key words: giant magnetostrictive film, piezoelectric ceramics, composite sensor, chaos

The nonlinear magnetoelectric response characteristics of a giant magnetostrictivepiezoelectric composite sensor in harmonic magnetic fields are described in this paper. Nonlinear differential items are introduced to explain the hysteresis phenomena of a giant magnetostrictive material. The nonlinear dynamic model of giant magnetostrictivepiezoelectric composite sensors in harmonic magnetic fields is developed, and the dynamic response of the system is obtained. The numerical and experimental results show that there are multiple frequencies in the response of the system, and the system's motions change from periodic orbits to chaos with the increase in the level of outside excitation. The results of this study are helpful for the optimal design and improvement of giant magnetostrictive-piezoelectric composite sensors.

\section{Introduction}

The giant magnetostrictive-piezoelectric composite sensor is a type of novel magnetoelectric sensor used to measure magnetic field intensity (MFI). It is highly sensitive, simple in structure, low in cost, and can be used to measure low-intensity magnetic fields. Many researchers have studied magnetostrictive-piezoelectric laminate composites. Ryu et al. were the first to study the magnetoelectric properties of piezoelectric and magnetostrictive laminate composites. ${ }^{(1)}$ Nan and Li calculated the giant magnetoelectric effects in ferroic composites of rare-earth-iron alloys and ferroelectric polymers. ${ }^{(2)}$ Dong et al. analyzed longitudinal and transverse magnetoelectric voltage coefficients of magnetostrictive piezoelectric laminate composites. ${ }^{(3)}$ Nersessian et al. studied the magnetoelectric behavior of Terfenol-D composite and lead zirconate titanate (PZT) ceramic laminates. ${ }^{(4)}$ Fillippov proposed the theory of the magnetoelectric effect in ferromagnetic-piezoelectric heterostrucures. ${ }^{(5)} \mathrm{Li}$ et al. designed a type of highsensitivity passive magnetic transducer based on PZT plates and a Fe-Ni fork substrate. ${ }^{(6)}$ Although many achievements on the magnetostrictive-piezoelectric composite structure were obtained in previous years, the theoretical results on the dynamic characteristics

${ }^{*}$ Corresponding author: e-mail: zhuzhiwen@tju.edu.cn 
of the magnetostrictive-piezoelectric composite sensors are few owing to their complex nonlinear characteristics. ${ }^{(7-12)}$

In this paper, we aim to introduce a method of analyzing the nonlinear magnetoelectric response characteristics of giant magnetostrictive-piezoelectric composite sensors in harmonic magnetic fields. Van der Pol items are introduced to explain the hysteresis phenomena of Terfenol-D. The nonlinear dynamic model of giant magnetostrictive-piezoelectric composite sensors in harmonic magnetic fields is developed, and the nonlinear dynamic responses of the system are obtained. Finally, the nonlinear dynamic characteristics of the system are given by simulation and experiment.

\section{Nonlinear Dynamic Model of Giant Magnetostrictive-Piezoelectric Sensors}

The structure of giant magnetostrictive-piezoelectric composite sensors is shown in Fig. 1. The composite sensor is regarded as a composite cantilever beam. It is made of Terfenol-D film, polyimide (PI) substrate and PZT piezoelectric ceramics. To analyze the dynamic characteristics of the composite sensors, constitutive models of Terfenol-D and piezoelectric ceramics should be obtained. The magnetic field intensity (MFI)-strain curve of Terfenol-D is shown in Fig. 2.

There are hysteretic phenomena in the MFI-strain curves of Terfenol-D. In this paper, the Van der Pol hysteretic model is introduced to describe the hysteretic nonlinear characteristics of Terfenol-D's MFI-strain curves. The Van der Pol equation is a nonlinear equation, as follows.

$$
\ddot{x}+x+\left(1-x^{2}\right) \dot{x}=0
$$

The item $\left(1-x^{2}\right) \dot{x}$ is called the Van der Pol item, which is symmetrical about the original point $(0,0)$. In this paper, the Van der Pol hysteretic model is developed to describe the Terfenol-D's MFI-strain curves as

$$
\varepsilon=e_{1} H+\left(e_{2} H-e_{3} H^{2}\right) \dot{H},
$$

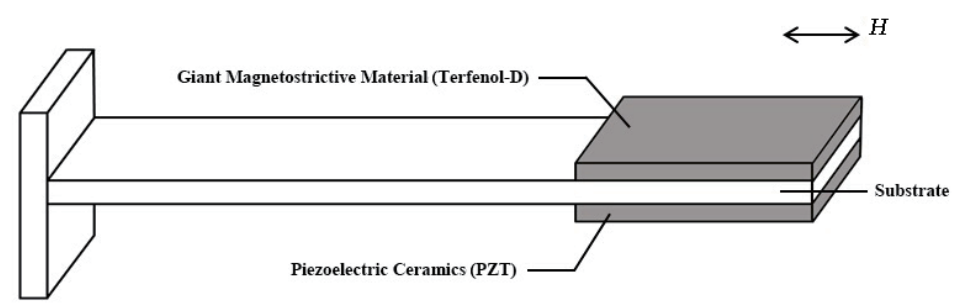

Fig. 1. Structure of magnetostrictive-piezoelectric composite sensors. 


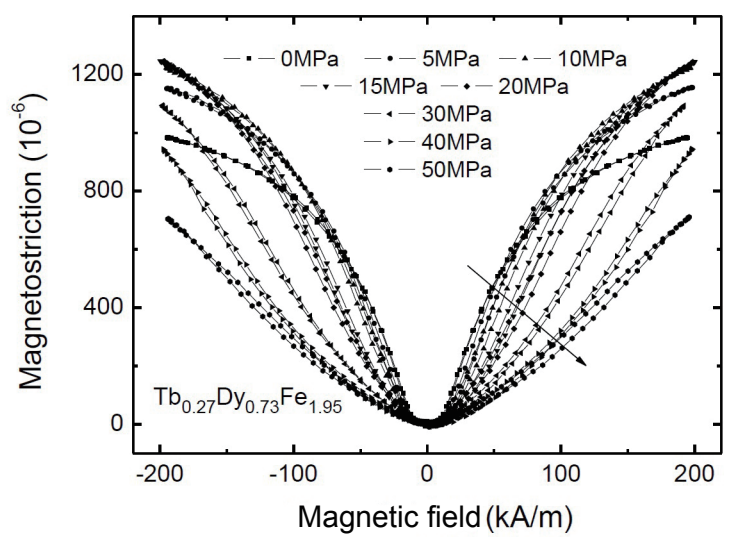

Fig. 2. MFI-strain curves of Terfenol-D.

where $\varepsilon$ is the strain, $H$ is the MFI, and $e_{i}(i=1-3)$ are the coefficients that are determined by the hysteretic loop.

The displacement-voltage curves of PZT are shown in Fig. 3. Similar to Terfenol-D, the hysteretic nonlinear piezoelectric equation of PZT can be shown as

$$
D=\varepsilon_{33}^{\mathrm{s}} E_{3}+k_{1} E_{3}^{3}+\left(k_{2} E_{3}-k_{3} E_{3}^{2}\right) \dot{E}_{3},
$$

where $D$ is the electric displacement, $E_{3}$ is the electric field intensity, $\varepsilon_{33}^{\mathrm{s}}$ is the dielectric coefficient and $k_{i}(i=1,2)$ are the coefficients that are determined using the hysteretic loop.

In this paper, Hamilton's principle is introduced to the dynamic modeling of the system. Hamilton's principle can be presented as

where

$$
\delta S=\int_{t_{1}}^{t_{2}} \delta\left(T_{1}+T_{2}-U_{1}-U_{2}-M_{\mathrm{P}}+W_{\mathrm{H}}+W_{\mathrm{d}}\right) d t=0,
$$

$$
\begin{gathered}
T_{1}=\frac{1}{2} \int_{0}^{l} \rho_{1} A_{1}\left(\frac{\partial u}{\partial t}\right)^{2} d x, T_{2}=\frac{1}{2} \int_{0}^{L} \rho_{2} A_{2}\left(\frac{\partial u}{\partial t}\right)^{2} d x \\
U_{1}=\frac{1}{2} \int_{0}^{l} \frac{E_{1} I_{1}}{2}\left(\frac{\partial^{2} u}{\partial x^{2}}\right)^{2} d x+\frac{E_{1} A_{1}}{8 l}\left[\int_{0}^{l} X_{1}\left(\frac{\partial u}{\partial x}\right)^{2} d x\right]^{2} \\
U_{2}=\frac{1}{2} E_{2} A_{2} \int_{0}^{L}\left[\left(\frac{\partial u}{\partial x}\right)^{2}+b_{1}\left(\frac{\partial u}{\partial x}\right)^{4}\right] d x, M_{\mathrm{P}}=\frac{1}{2} \iiint E_{3} D d V=\frac{A_{1}}{2} \int_{0}^{l} E_{3} D d x \\
W_{H}=\int_{0}^{l} \sigma A d x=\int_{0}^{l} E A\left[e_{1} H+\left(e_{2} H-e_{3} H^{2}\right) \dot{H}\right] d x, W_{\mathrm{d}}=\int_{0}^{L}-c u \frac{\partial u}{\partial t} d x
\end{gathered}
$$




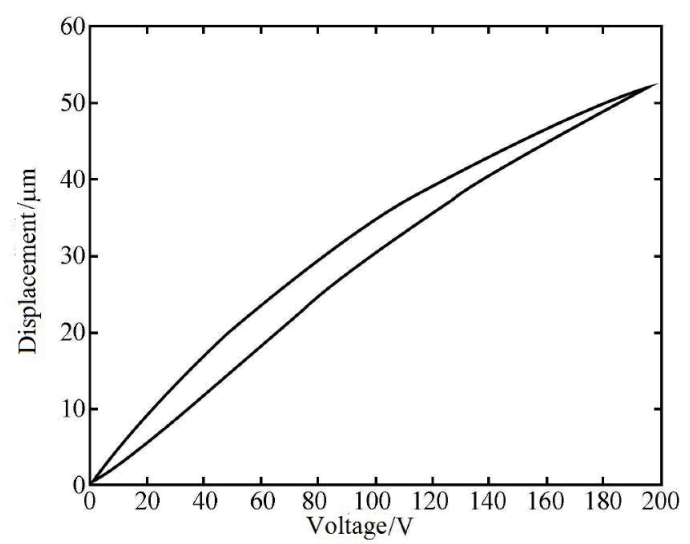

Fig. 3. Displacement-voltage curves of PZT.

Here, the kinetic energy and potential energy of the Terfenol-D film are ignored since the thickness of the Terfenol-D film is only $50 \mathrm{~nm}$; $l$ is the length of the Terfenol-D film and PZT piece; $L$ is the length of the substrate; $T_{1}$ is the kinetic energy of PZT; $T_{2}$ is the kinetic energy of the substrate; $U_{1}$ is the potential energy of PZT; $U_{2}$ is the potential energy of the substrate; $u=u(x, t)$ is the vibration mode of the cantilever beam; $E_{i}(i=1,2)$, $\rho_{i}(i=1,2)$, and $A_{i}(i=1,2)$ are the elastic modulus, density, and cross-sectional area of the materials, respectively; 1 : PZT, 2: PI substrate; $M_{\mathrm{P}}$ is the electric field energy of PZT; $W_{H}$ is the work done by the magnetic field; $W_{\mathrm{d}}$ is the work done by the system's damp force.

Thus, we obtain

$$
m \frac{\partial^{2} u}{\partial t^{2}}+\left(c+\int_{0}^{l} E_{2} A \varepsilon d x\right) \frac{\partial u}{\partial t}-a_{1} \frac{\partial^{2} u}{\partial x^{2}}-a_{2} \frac{\partial^{4} u}{\partial x^{4}}-a_{3} \int_{0}^{L} E_{3} D d x=\frac{1}{2}\left(E_{2} A_{2} \frac{\partial^{2} u}{\partial x^{2}}+\rho_{2} A_{2} \frac{\partial^{2} u}{\partial t^{2}}\right),
$$

where $m=\rho_{1} A_{1}+\frac{1}{3} \rho_{2} A_{2}, a_{1}=\frac{E_{2} A_{2}}{3}+\frac{\varepsilon_{33}^{\mathrm{s}} E_{1} A_{1}}{2}, a_{2}=\frac{k_{1} A_{2}}{8 L}$, and $a_{3}=\frac{A_{1}}{4 L}$.

For cantilever composite sensors, their vibration mode is $u(x, t)=\psi(x, t) y(t)$, where $\psi(x)=\sin \lambda_{i} x-\sinh \lambda_{i} x+\bar{\alpha}_{i}\left(\cosh \lambda_{i} x-\cos \lambda_{i} x\right), y=y(t)$ is the amplitude of system response, $\cos \lambda_{i} L \cosh \lambda_{i} L+1=0, \bar{\alpha}_{i}=\frac{\sinh \lambda_{i} L+\sin \lambda_{i} L}{\cosh \lambda_{i} L+\cos \lambda_{i} L}$. For the harmonic magnetic field, $H=\bar{H} \cos \omega t$, where $\bar{H}$ and $\omega$ are the intensity and frequency of the harmonic magnetic field, respectively. Thus, the dynamic equation of system response can be solved from eq. (5) by Galerkin's method as

$$
\ddot{y}+2 \eta \dot{y}+c_{1} y+c_{2} y^{3}+\left(c_{3} y-c_{4} y^{2}\right) \dot{y}=b \cos \omega t,
$$

where $2 \eta=\frac{c}{m}, c_{1}=\frac{4 \pi^{2} a_{1} L^{2}-\pi^{4} a_{2}}{16 m L^{4}}, c_{2}=\frac{\pi^{4} a_{3}}{32 m L^{3}}, c_{3}=\frac{a_{3} k_{2}}{4 m L^{2}}, c_{4}=\frac{a_{3} k_{3}}{8 m L^{3}}$, and $b=\frac{\bar{H}}{4 m L^{2}}$. 


\section{Dynamic Characteristics of the System}

The approximate solution of eq. (6) can be shown as

$$
y=a \cos (\omega t+\theta)+\frac{c_{2} a^{3}}{32 \omega^{2}} \cos [3(\omega t+\theta)]+\frac{c_{3} a^{2}}{8 \omega^{2}} \sin [2(\omega t+\theta)]-\frac{c_{4} a^{3}}{32 \omega^{2}} \sin [3(\omega t+\theta)] .
$$

The numerical results of the system response and phase diagram are presented in Fig. 4 , where $\eta=0.02, c_{1}=2500, c_{2}=100, c_{3}=10, c_{4}=0.7$, and $\omega=30 \mathrm{~Hz}$. The numerical results indicate that there are multiple frequencies in the dynamic response of the system, and the system's motions change from periodic orbits to chaos with the increase in the level of outside excitation. The multiple frequency response is induced by the hysteretic nonlinear characteristics of Terfenol-D and PZT. To eliminate the effect of the multiple frequencies, the sensor's materials should be as linear as possible. It also means that a nonlinear compensation technology should be applied in giant magnetostrictivepiezoelectric composite sensors to extend their measurement range.

The experimental results of giant magnetostrictive-piezoelectric composite sensors subjected to harmonic magnetic excitation are shown in Figs. 5-7. We can see that the experimental results are in agreement with the theoretical and numerical results.

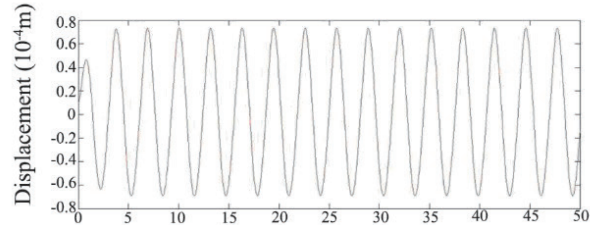

(a)

Time (s)

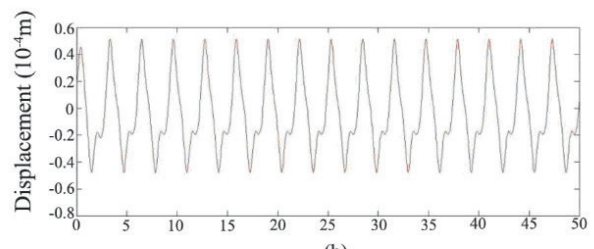

(b)

Time (s)

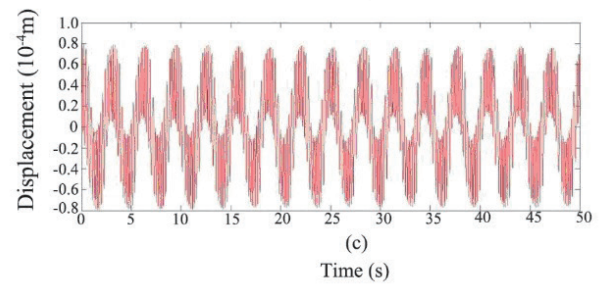

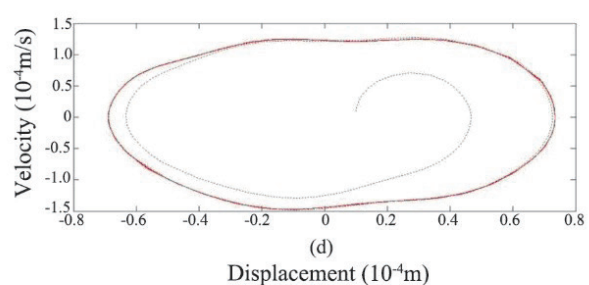

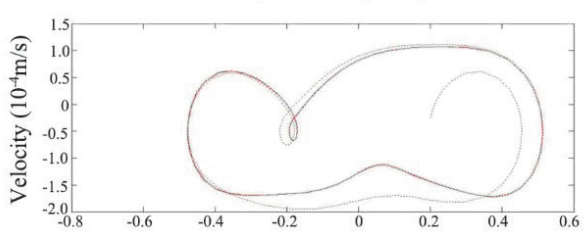

(e)

Displacement $\left(10^{-4} \mathrm{~m}\right)$

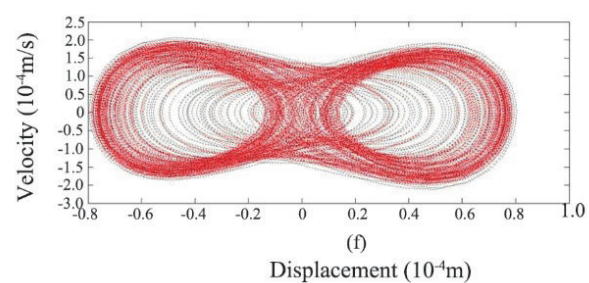

Fig. 4. (Color online) Time course and phase diagram of the system. (a, d) $b=5$; (b, e) $b=10$; and (c, f) $b=20$. 


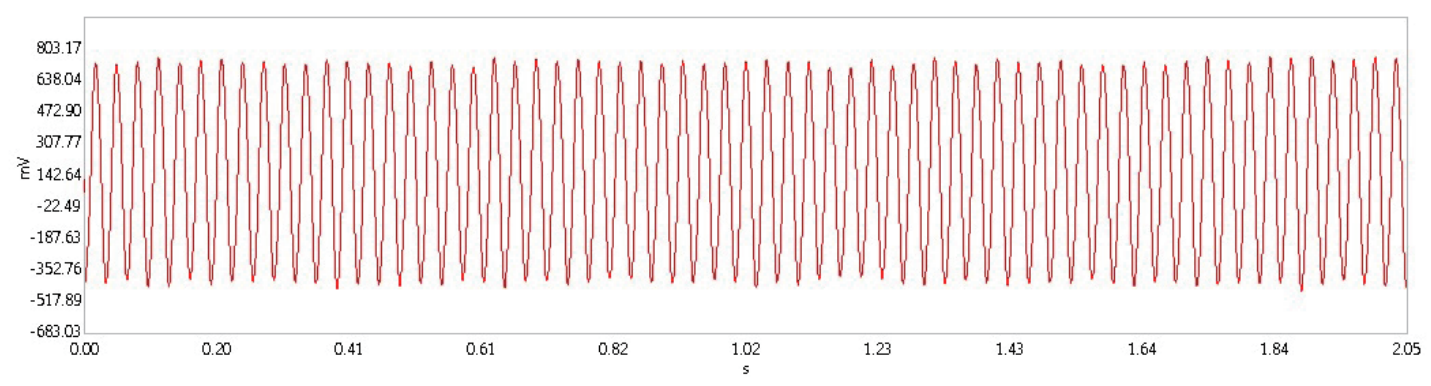

Fig. 5. (Color online) Output voltage of the composite sensor when $\bar{H}=20 \mathrm{kA} / \mathrm{m}$.

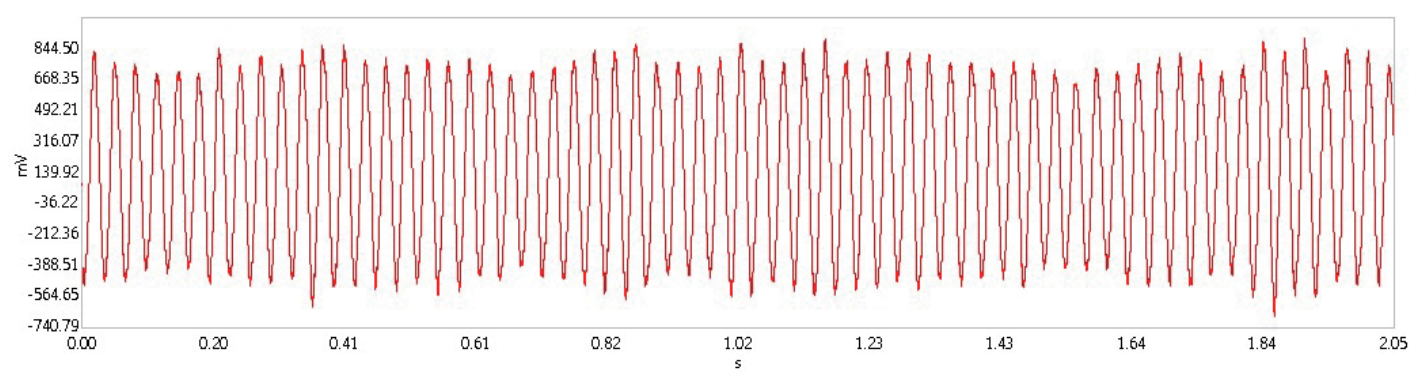

Fig. 6. (Color online) Output voltage of the composite sensor when $\bar{H}=60 \mathrm{kA} / \mathrm{m}$.

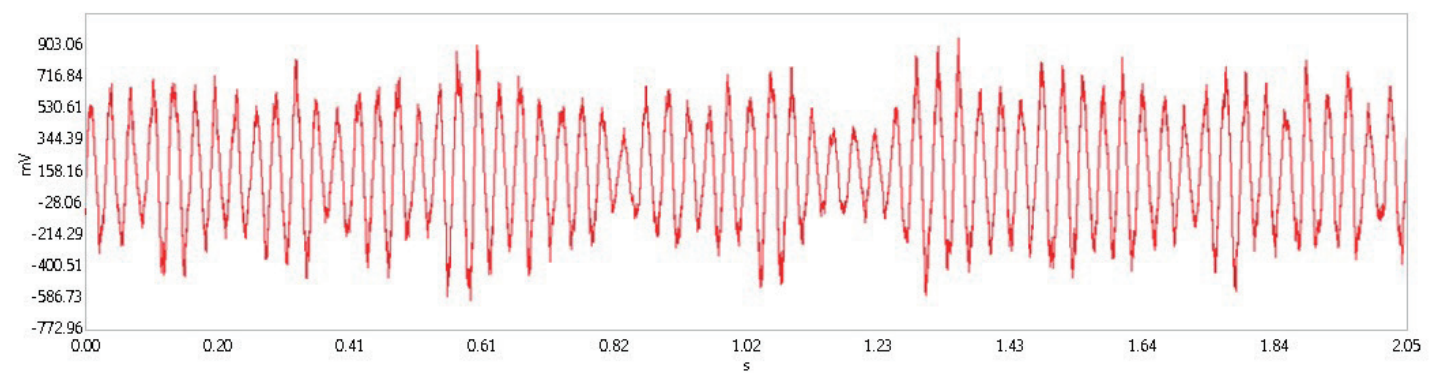

Fig. 7. (Color online) Output voltage of the composite sensor when $\bar{H}=100 \mathrm{kA} / \mathrm{m}$.

\section{Conclusions}

The nonlinear magnetoelectric response characteristics of a giant magnetostrictivepiezoelectric composite sensor in harmonic magnetic fields are described in this paper. Van der Pol items are introduced to set up the nonlinear hysteretic models of Terfenol-D and PZT. The new models have a simple form and are easy to analyze. The nonlinear dynamic model of giant magnetostrictive-piezoelectric composite sensors in harmonic magnetic fields is developed, and the system's dynamic response is obtained. The 
numerical and experimental results show that there are multiple frequencies in the response of the system, and the system's motions change from periodic orbits to chaos with the increase in the level of outside excitation. The multiple frequency response is induced by the hysteretic nonlinear characteristics of Terfenol-D and PZT. To eliminate the effect of the multiple frequencies, the sensor's materials should be as linear as possible. It also means that nonlinear compensation technology should be applied in giant magnetostrictive-piezoelectric composite sensors to extend their measurement range. This paper's results are helpful for the optimal design and improvement of giant magnetostrictive-piezoelectric composite sensors.

\section{Acknowledgements}

The authors gratefully acknowledge the support of the Natural Science Foundation of China (NSFC) through Grant Nos. 11272229, 11302144, and 11402168, the Ph.D. Programs Foundation of the Ministry of Education of China through Grant No. 20120032120006, and the Tianjin Research Program of Application Foundation and Advanced Technology through Grant Nos. 13JCYBJC17900 and 14JCQNJC05300.

\section{References}

1 J. Ryu, A. V. Carazo and K. Uchino: J. Appl. Phys. 40 (2001) 4948.

2 C. W. Nan and M. Li: Phys. Rev. B 63 (2001) 144415.

3 S. X. Dong, J. F. Li and D. Viehland: IEEE Trans. Ultrason. Freroelectr. Freq. Control 50 (2003) 1253.

4 N. Nersessian, S. W. Or and G. P. Carman: IEEE Trans. Magn. 40 (2004) 2646.

5 D. A. Fillippov: Phys. Solid State 47 (2005) 1080.

6 P. Li, Y. M. Wen and L. X. Bian: Appl. Phys. Lett. 90 (2007) 022503.

7 V. M. Petrov, G. Srinivasan and M. I. Bichurin: Phys. Rev. B 75 (2007) 224407.

8 C. S. Lee, J. Joo and S. Han: Appl. Phys. Lett. 85 (2004) 1841.

9 Z. Y. Jia, W. Liu and Y. S. Zhang: Sens. Actuators, A 128 (2006) 158.

10 Q. X. Yang, H. Y. Chen and S. Z. Liu: IEEE Trans. Magn. 42 (2006) 939.

11 S. Masuda, Y. Matsumura and Y. Nishi: J. Jpn. Inst. Met. 70 (2006) 166.

12 N. Tiercelin, V. Preobrazhensky and P. Pernod: J. Magn. Magn. Mater. 210 (2000) 302. 